\title{
Right heart catheterisation: best practice and pitfalls in pulmonary hypertension
}

\author{
Stephan Rosenkranz ${ }^{1}$ and loana R. Preston ${ }^{2}$
}

Affiliations: ${ }^{1}$ Dept III of Internal Medicine and Cologne Cardiovascular Research Center (CCRC), Cologne University Heart Center, Cologne, Germany. ${ }^{2}$ Pulmonary, Critical Care, and Sleep Division, Tufts Medical Center, Boston, MA, USA.

Correspondence: Stephan Rosenkranz, Dept III of Internal Medicine, Cologne University Heart Center, Kerpener Str. 62, Cologne 50937, Germany. E-mail: stephan.rosenkranzQuk-koeln.de

ABSTRACT Right heart catheterisation (RHC) plays a central role in identifying pulmonary hypertension $(\mathrm{PH})$ disorders, and is required to definitively diagnose pulmonary arterial hypertension (PAH). Despite widespread acceptance, there is a lack of guidance regarding the best practice for performing RHC in clinical practice. In order to ensure the correct evaluation of haemodynamic parameters directly measured or calculated from RHC, attention should be drawn to standardising procedures such as the position of the pressure transducer and catheter balloon inflation volume. Measurement of pulmonary arterial wedge pressure, in particular, is vulnerable to over- or under-wedging, which can give rise to false readings. In turn, errors in RHC measurement and data interpretation can complicate the differentiation of PAH from other PH disorders and lead to misdiagnosis. In addition to diagnosis, the role of RHC in conjunction with noninvasive tests is widening rapidly to encompass monitoring of treatment response and establishing prognosis of patients diagnosed with PAH. However, further standardisation of RHC is warranted to ensure optimal use in routine clinical practice.

@ERSpublications

Right heart catheterisation is required to diagnose pulmonary arterial hypertension, but standardisation is needed http://ow.ly/TI6Gr

\section{Introduction}

Clinical experience with right heart catheterisation (RHC) can be charted from its first application in a self-catheterisation feasibility experiment by Dr Forssmann in the 1920s, to the introduction of the Swan-Ganz balloon floatation catheter in 1970 for immediate bedside monitoring $[1,2]$. Knowledge gained with RHC in the clinic beyond those early years has greatly enhanced understanding of the haemodynamic impairment that results from various clinical conditions and established its central role in the diagnosis of pulmonary vascular disorders [1-3].

Today, RHC is the diagnostic gold standard for pulmonary hypertension (PH), a serious condition defined by a mean pulmonary arterial pressure (PAP) $\geqslant 25 \mathrm{mmHg}$ at rest $[3,4]$. There are five subgroups of $\mathrm{PH}$, encompassing pulmonary arterial hypertension (PAH) (group 1); $\mathrm{PH}$ due to left heart disease (group 2); $\mathrm{PH}$ due to lung diseases and/or hypoxia (group 3); chronic thromboembolic pulmonary hypertension (group 4); and $\mathrm{PH}$ with unclear multifactorial mechanisms (group 5) [5]. RHC is also used to further classify these groups into pre-capillary (groups 1, 3, 4 and 5) or post-capillary (groups 2 and 5) $\mathrm{PH}$ populations on the basis of a pulmonary arterial wedge pressure (PAWP) threshold of $15 \mathrm{mmHg}$ [4]. In fact, RHC is the definitive diagnostic technique for reliably confirming whether a patient has PAH [3]. Characterised by the presence of pre-capillary $\mathrm{PH}, \mathrm{PAH}$ is determined by a PAWP $\leqslant 15 \mathrm{mmHg}$ in addition to a mean PAP $\geqslant 25 \mathrm{mmHg}$ at rest and a pulmonary vascular resistance (PVR) $>3$ Wood units [3].

Received: Aug 212015 | Accepted after revision: Oct 142015

Conflict of interest: Disclosures can be found alongside the online version of this article at err.ersjournals.com

Provenance: Publication of this peer-reviewed article was sponsored by Actelion Pharmaceuticals Ltd, Allschwil, Switzerland (principal sponsor, European Respiratory Review issue 138).

Copyright CERS 2015. ERR articles are open access and distributed under the terms of the Creative Commons Attribution Non-Commercial Licence 4.0. 
In addition to its use in diagnosis, RHC provides useful information on the degree of haemodynamic impairment, determines response to $\mathrm{PAH}$ therapy and establishes prognosis, thereby informing clinical decision-making in the management of PAH $[4,6]$. However, as shown in the RePHerral study of newly diagnosed patients with $\mathrm{PH}$ referred to an expert centre, not all patients receive RHC as part of their diagnostic work-up [7]. Reasons for not performing RHC may include lack of knowledge or training, cost and the perception of risk associated with the invasive nature of RHC [8]. While RHC is not always performed, it remains the gold standard for diagnosing PAH. However, although there are numerous reviews in the literature on RHC, few focus on practical guidance for performing the procedure $[3,9]$.

In this article, we review best practice for the use of RHC in PAH diagnosis, discuss general pitfalls of RHC measurements and their consequences, and conclude with an overview of the widening application of RHC in the clinical setting.

\section{Best practice}

The diagnostic algorithm for PAH, updated by the joint task force of the European Society of Cardiology (ESC) and the European Respiratory Society (ERS) highlights the central role that RHC plays in correctly diagnosing PAH (fig. 1) [4]. The guidelines recommend that patients with unexplained exertional dyspnoea, syncope and/or signs of right ventricular dysfunction should be assessed for suspected $\mathrm{PH} / \mathrm{PAH}$ using transthoracic echocardiography (which remains the most widely used screening tool), with a confirmed diagnosis ultimately dependent on haemodynamic results obtained from RHC $[3,4,10]$.

\section{Haemodynamic parameters}

Practical recommendations for haemodynamic variables measured by RHC, which are either directly measured or calculated from the observed values, are summarised in table 1.

In the diagnostic work-up for $\mathrm{PAH}$, it is recommended that the RHC should include a comprehensive haemodynamic assessment comprising the measurement of cardiac output, mixed venous oxygen saturation $\left(\mathrm{S}_{\mathrm{vO}}\right)$, PAP, PAWP, right atrial pressure (RAP) and right ventricular pressure (table 1) [4]. Parameters calculated from these measurements include the transpulmonary pressure gradient, diastolic pressure gradient, PVR and cardiac index (table 1).

\section{Pressure transducer and zeroing}

One aspect of best practice guidance relates to the position of the pressure transducer, which is important for correct pressure measurements and has shown variation in zero levelling between centres [3]. To establish uniformity of the pressure transducer setting, the pressure transducer should be set to zero level at the mid-thoracic line (with a suggested reference point defined by the intersection of the frontal plane at the mid-thoracic level, the transverse plane at the level of fourth anterior intercostal space, and the midsagittal plane) with the patient in a supine position, halfway between the anterior sternum and bed surface, which represents the level of the left atrium (fig. 2) [4, 20].

\section{PAWP measurement}

The term PAWP is used interchangeably with pulmonary capillary wedge pressure and pulmonary artery occlusion pressure in the general literature [3]. However, consistent with recent recommendations [3], PAWP will be used throughout this article. When measuring PAWP, it is advised that the balloon should be inflated in the right atrium, from where the catheter should be advanced until it reaches the PAWP position [3]. In particular, repeated inflations and deflations in the wedge position should be avoided due to the risk of pulmonary artery rupture [3]. PAWP should be subsequently recorded as the mean of three measurements at end expiration [3].

The recommendation for end-expiratory measurement arises from the observation that evaluation of PAWP should be performed at functional residual volume, when the intra- and extra-thoracic pressures are equal. PAWP may be significantly affected by respiratory swings, which affect intrathoracic pressure. As these variations are minimal at the end of normal expiration, measurement of end-expiratory PAWP minimises this effect (fig. 3) [3]. Practical recommendations on handling respiratory swings are consistent with those in a recent comprehensive review, which reached the following key conclusions: there is a minimal influence of elastic lung recoil on pulmonary pressure readings at functional residual capacity (end expiration) and larger effects of intrathoracic pressure changes are observed in patients with chronic obstructive airway disease or those who are significantly obese $[14,20]$. In fact, all pressure measurements should be carefully recorded at end expiration, ensuring that the patient does not produce a Valsalva manoeuvre, which would affect the values. Furthermore, it should be noted that averaging PAWP over several respiratory cycles may be the most reasonable compromise to compensate for respiratory fluctuations as positive expiratory and negative inspiratory intrathoracic pressures cancel each other out [20]. 


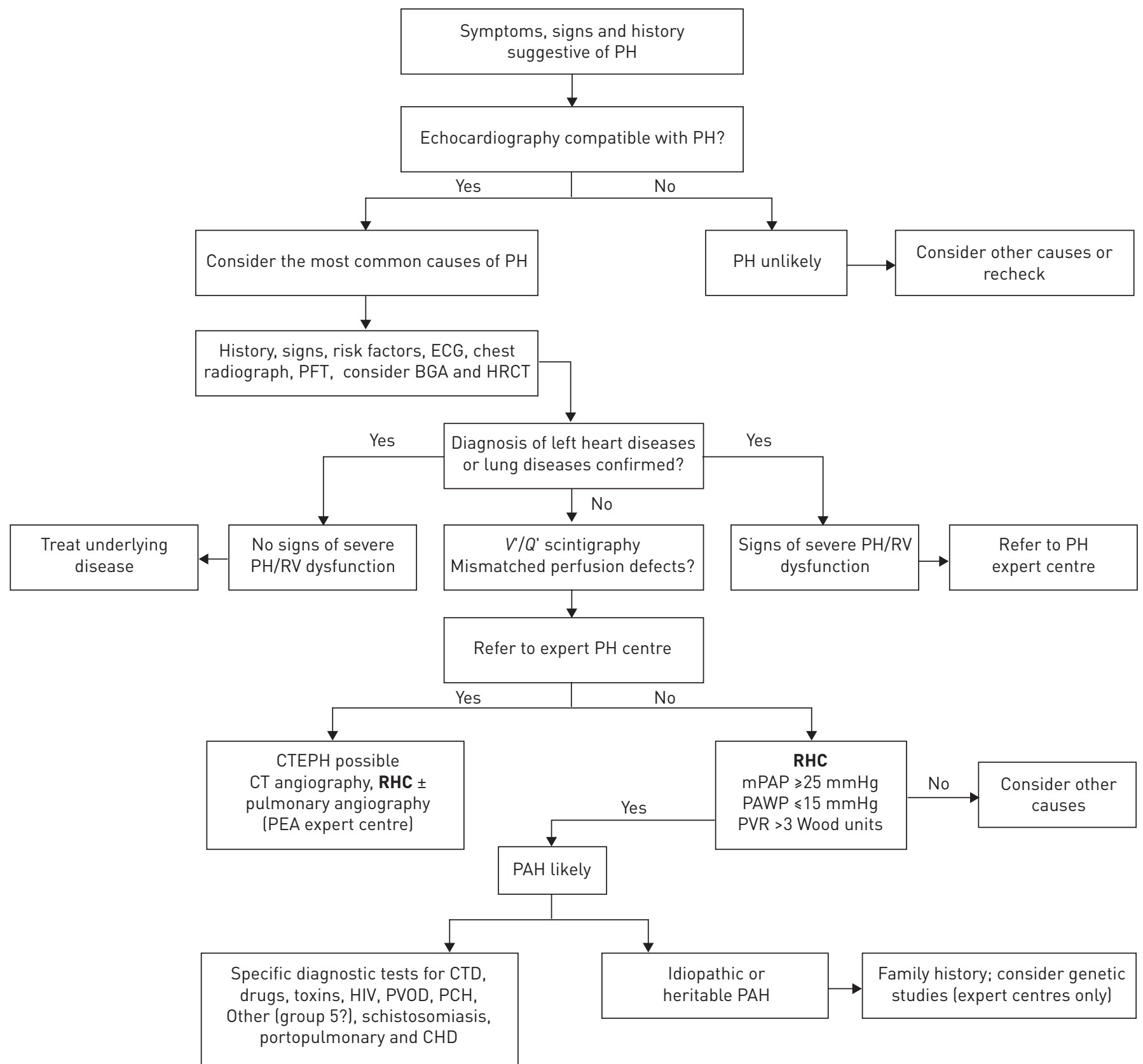

FIGURE 1 Diagnostic algorithm for pulmonary arterial hypertension (PAH). PH: pulmonary hypertension; PFT: pulmonary function testing; BGA: blood gas analysis; HRCT: high-resolution computed tomography; RV: right ventricular; $V^{\prime} / Q^{\prime}$ : ventilation/perfusion; CTEPH: chronic thromboembolic pulmonary hypertension; CT: computed tomography; RHC: right heart catheterisation; PEA: pulmonary endarterectomy; mPAP: mean pulmonary arterial pressure; PAWP: pulmonary arterial wedge pressure; PVR: pulmonary vascular resistance; CTD: connective tissue disease; PVOD: pulmonary veno-occlusive disease; PCH: pulmonary capillary haemangiomatosis; CHD: congenital heart disease. Reproduced and modified from [4] with permission from the publisher.

\section{Cardiac output}

Aside from pulmonary pressures, RHC enables the measurement of cardiac output using the widely established thermodilution method [21], which consists of a thermistor-tipped Swan-Ganz catheter that records the decrease in blood temperature in the pulmonary artery after injection of $10 \mathrm{~mL}$ of normal saline (or another indicator) at room temperature. The change in blood temperature is inversely proportional to the dilution of the injectate and therefore enables derivation of computer-generated cardiac output values [21]. The thermodilution technique was cited as the preferred method of cardiac output monitoring by the European Society of Cardiology (ESC)/European Respiratory Society (ERS) guidelines for the diagnosis and treatment of pulmonary hypertension, even among patients with very low cardiac output and/or severe 
TABLE 1 Practical recommendations relating to parameters measured or derived from right heart catheterisation

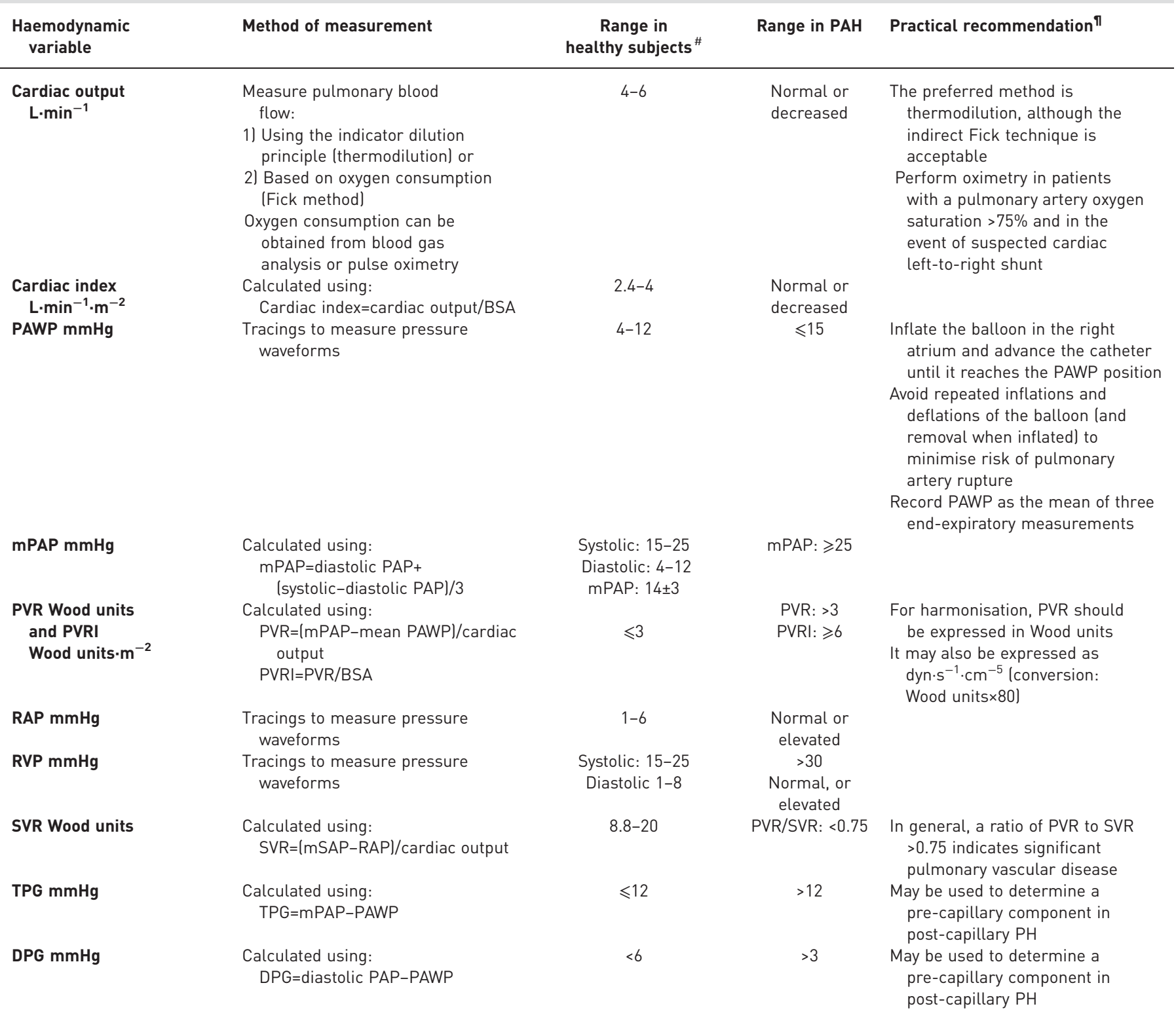

PAH: pulmonary arterial hypertension; PAWP: pulmonary arterial wedge pressure; PAP: pulmonary arterial pressure; PVR: pulmonary vascular resistance; PVRI: pulmonary vascular resistance index; RAP: right atrial pressure; RVP: right ventricular pressure; SVR: systemic vascular resistance; TPG: transpulmonary pressure gradient; DPG: diastolic pressure gradient; BSA: body surface area; mPAP: mean pulmonary arterial pressure; mSAP: mean systemic arterial pressure; PH: pulmonary hypertension. \#: values for PVR and PAP have been identified in additional studies of healthy subjects $[18,19]$; ${ }^{\text {I: }}$ information for practical recommendations from $[3,15,17]$. Information from $[3,11-19]$.

tricuspid regurgitation [4]. The thermodilution technique has proven to be a reliable method when compared with the direct Fick measurement (which requires a directly obtained measurement of oxygen uptake) [3].

The indirect Fick technique, which estimates the amount of oxygen uptake, may also be employed for determination of cardiac output. For this purpose, $\mathrm{SvO}_{2}$ is measured while the tip of the catheter is in the right atrium or pulmonary artery, whereas systemic oxygen saturation is often acquired noninvasively by oximetry [14]. The indirect Fick method is less reliable than the other techniques and is therefore not the preferred method for the measurement of cardiac output [3].

In addition to evaluation of cardiac output, the cardiac index should be calculated as the ratio of cardiac output to body surface area [12] (table 1). Although cardiac output varies widely depending on the patient's weight and size, a normal cardiac index value always ranges between 2.4 and $4.0 \mathrm{~L} \cdot \mathrm{min}^{-1} \cdot \mathrm{m}^{-2}[16,20]$. 


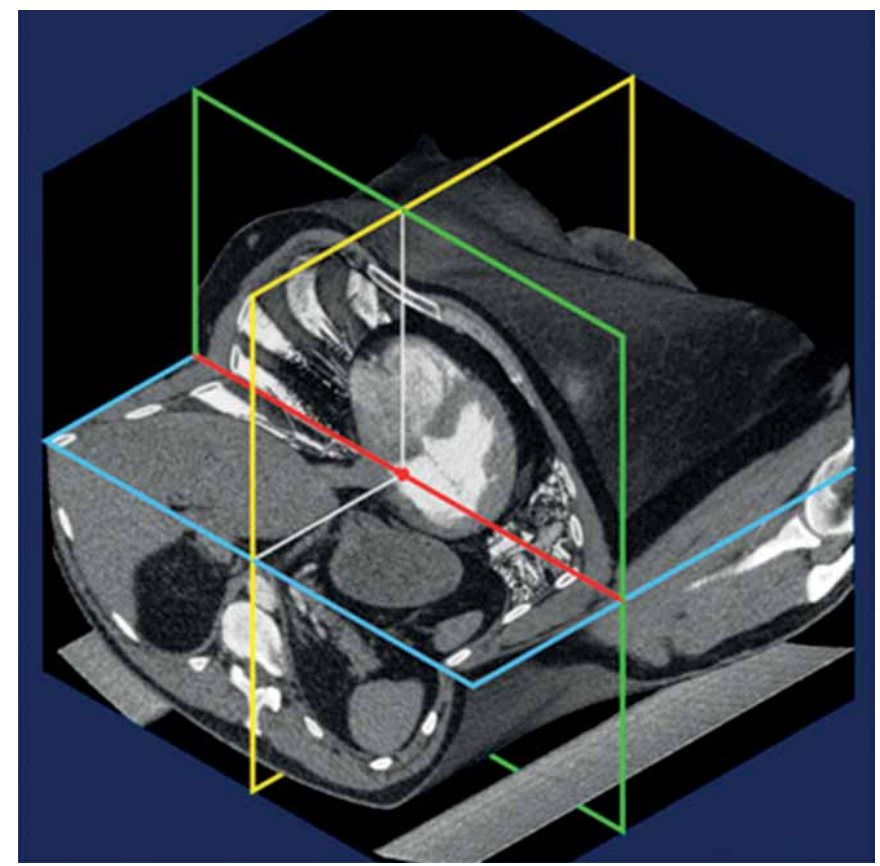

FIGURE 2 Best practice recommendations for right heart catheterisation: pressure transducer and zeroing $[3,20]$. The joint task force of the European Society of Cardiology and the European Respiratory Society recommends setting the pressure transducer to zero at the mid-thoracic line (with a suggested reference point defined by the intersection of the frontal plane at the mid-thoracic level, the transverse plane at the level of fourth anterior intercostal space, and the midsagittal plane [20]) in a supine patient halfway between the anterior sternum and the bed surface [4]. Reproduced from [20] with permission from the publisher.
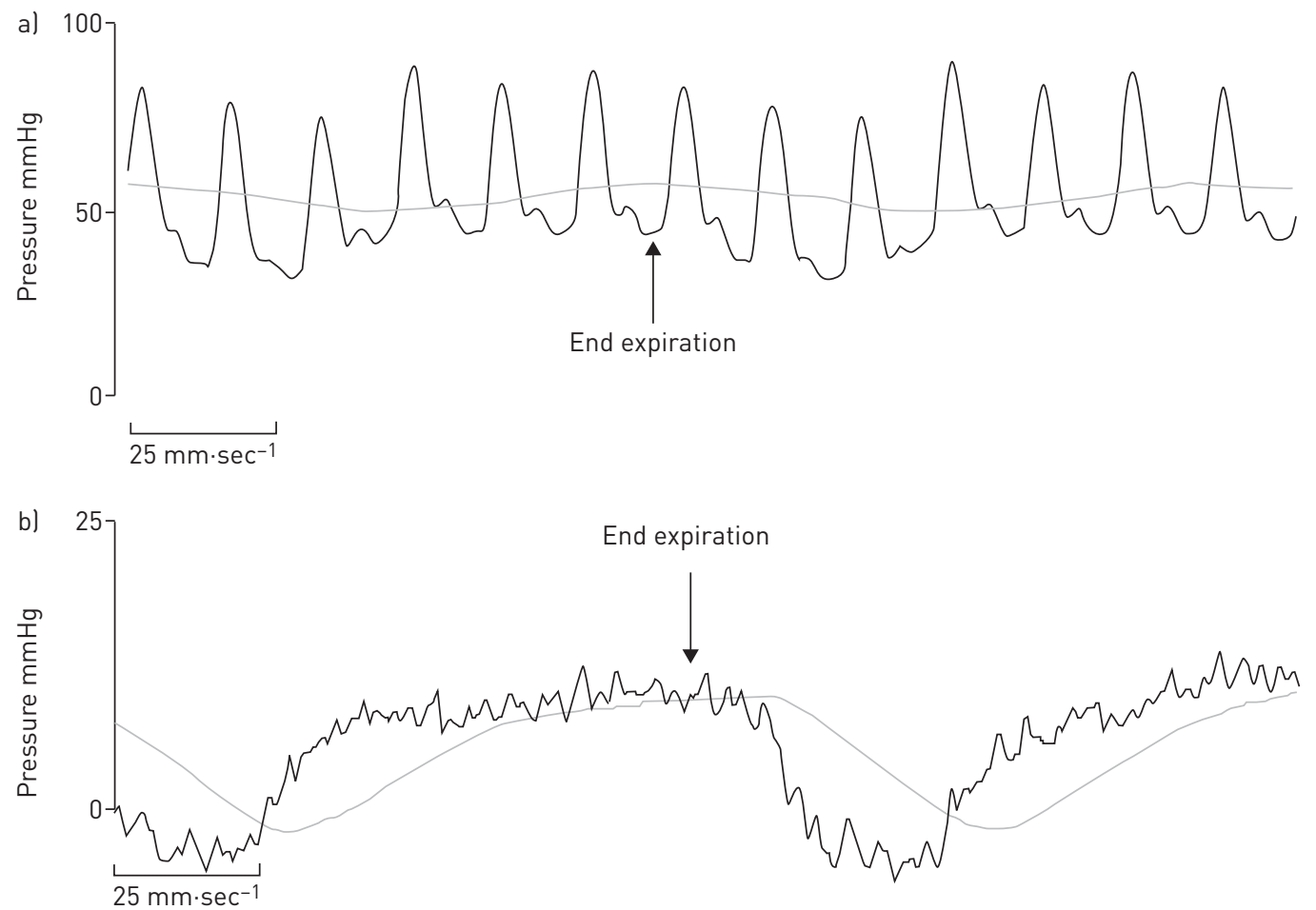

FIGURE 3 Representative pressure tracings of a) pulmonary arterial pressure and b) pulmonary arterial wedge pressure (PAWP). Both recordings display respiratory variations, and the correct measurements should be made at the end of the expiratory phase (arrows). For measurement of PAWP (b), the digital read is usually obtained automatically and averages over the inspiration and expiration, with a resultant value of $4 \mathrm{mmHg}$ The correct value is indicated by the arrow marking the end-expiratory phase when the respiratory system is at functional residual capacity and is $10 \mathrm{mmHg}$. 


\section{Shunt assessment}

High pulmonary artery oxygen saturation may indicate a cardiac left-to-right shunt [14]. Therefore, stepwise assessment of oxygen saturation by oximetry is recommended in patients with a pulmonary artery oxygen saturation $>75 \%$ and when a cardiac left-to-right shunt is suspected [3]. In this instance, measurements should include superior vena cava, inferior vena cava, right atrium high, right atrium middle, right atrium low, right ventricle and pulmonary artery saturations. A step-up in oxygen saturation of $\geqslant 7 \%$ may be indicative of an atrial left-to-right shunt, whereas $\geqslant 5 \%$ may signal a shunt at the level of the right ventricle or pulmonary artery [22]. When a left-to-right shunt is suspected, the direct Fick method is the preferred means of cardiac output measurement.

\section{Pulmonary vascular resistance}

The calculation of PVR and PVR index is crucial in establishing the diagnosis of PAH. These parameters are derived as the ratio of the transpulmonary pressure gradient to cardiac output (table 1). PVR has been incorporated into the ESC/ERS guidelines [4], with a value $>3$ Wood units forming part of the haemodynamic definition of PAH due to its relevance in determining prognosis $[3,23]$. Moreover, the ratio of PVR to systemic vascular resistance, calculated from arterial pressure and cardiac output, can be applied to indicate the severity of pulmonary vascular disease $[11,15]$.

\section{Vasoreactivity testing}

The use of vasoreactivity testing during RHC in patients with idiopathic, heritable or drug- and toxin-induced PAH helps to identify those likely to benefit from treatment with calcium channel blockers (CCBs) [4]. CCBs have been previously shown to elicit significant decreases in PAP and PVR in patients with PAH [24-26]. However, the therapeutic utility of long-term treatment with CCBs in patients other than those with idiopathic PAH is less clear [4]. It is recommended that patients with idiopathic PAH who are treated with CCBs after a positive vasodilator response should be closely monitored and reassessed after 3-4 months of therapy, including undertaking a RHC [4]. For other forms of PAH or PH, use of pulmonary vasoreactivity to evaluate potential response to CCB therapy is not recommended, as the results can be misleading and potentially fatal (if incorrect treatment decisions are made), and do not necessarily help in choosing a specific therapy $[3,27]$.

In summary, the diagnosis of PAH relies on the results from haemodynamic parameters directly measured or calculated from RHC. There are a number of best practice recommendations that should be followed when performing RHC to avoid errors in diagnosis and the risk of complications.

\section{Pitfalls associated with measurement errors}

Although RHC is an accepted diagnostic tool, the technique still lacks proper standardisation for the diagnosis of $\mathrm{PAH}$, rendering it vulnerable to procedural or data interpretation errors $[3,14]$. Errors in data acquisition may also result from the failure to appropriately maintain and calibrate technical equipment [14]. Therefore, incorrect application of the RHC technique can also impact the measured variables [14].

\section{Pitfalls in PAWP measurement}

PAWP is used to differentiate $\mathrm{PH}$ associated with left heart disease from pre-capillary $\mathrm{PH}$ conditions [28], but it is especially vulnerable to errors in measurement [29]. It is therefore critical to obtain the typical pressure tracing, which includes an a wave and a $\mathrm{v}$ wave [11]. The catheter balloon inflation volume, in particular, may affect the validity of PAWP measurement as a result of over- or under-wedging [14, 29]. Over-wedging, attributed to excessive inflation of the catheter balloon, can give rise to false high or low PAWP readings and the potentially fatal complication of pulmonary artery rupture [14]. The influence of over-wedging on PAWP was demonstrated in a study of 37 patients with suspected $\mathrm{PH}$ who underwent RHC [29]. Results from the study demonstrated the potential for false elevations in PAWP at full balloon inflation volume $(1.5 \mathrm{~mL}$ of air and balloon diameter of $1.3 \mathrm{~cm})$, whereas half inflation $(0.75 \mathrm{~mL}$ of air and diameter of $\sim 0.9 \mathrm{~cm}$ ) was shown to be safe and to correlate with greater precision and less bias in the observed values [29]. The more commonly observed under-wedging is frequently seen in patients with very high PAP, and arises from incomplete occlusion of the branch pulmonary artery by the catheter balloon, which leads to false elevation of PAWP values [14]. This most probably happens because the lumens of pulmonary arteries in severely ill patients taper off abruptly, making it difficult to achieve a tight seal with the balloon.

The use of end-expiratory PAWP, while recommended by the ESC/ERS guidelines, remains a subject of debate $[30,31]$. In a recent retrospective analysis of 329 spontaneously breathing patients who underwent RHC, tracings were evaluated to compare end-expiratory PAWP readings with those obtained when PAWP was averaged throughout the respiratory cycle [30]. Overall, 29\% of patients with a pre-capillary phenotype would have been misclassified as having post-capillary $\mathrm{PH}$, based on measurement of 


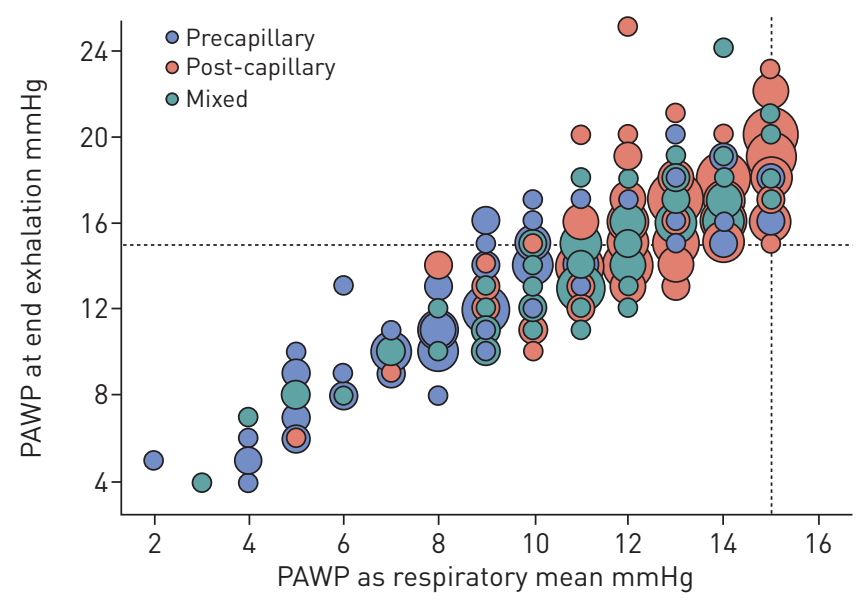

FIGURE 4 Potential for misclassification of pulmonary hypertension with reliance on end-expiratory pulmonary arterial wedge pressure (PAWP). Distribution of PAWP at end exhalation versus as calculated by the respiratory mean according to clinical phenotype. Larger circles represent identical observations in multiple patients. Blue circles above $15 \mathrm{mmHg}$ represent phenotypically pre-capillary patients who would have been misclassified as having pulmonary venous hypertension using end-expiratory PAWP. Reproduced from [30] with permission from the publisher.

end-expiratory PAWP (fig. 4) [30]. However, the end-expiratory method is supported by an earlier study, which showed that use of digitised PAWP (measured by the automated computer) instead of end-expiratory PAWP resulted in the misdiagnosis of nearly 30\% of $\mathrm{PH}$ group 2 patients as having $\mathrm{PAH}$ [31]. In addition, the use of diuretics may mask an elevated left ventricular end-diastolic pressure and PAWP in patients with left heart disease [32]. Regardless of the method used, physicians should be aware of potential misclassifications when interpreting their results and invasive haemodynamics should always be interpreted in the context of the clinical picture and non-invasive tests, particularly echocardiography.

\section{Pitfalls in measurement of cardiac output}

The measurement of cardiac output with RHC can also be affected by measurement errors. Specifically, applying the indirect Fick technique leads to a high likelihood of inaccuracy in cardiac output, as results are frequently based on estimated values (rather than direct measurements) for oxygen uptake due to the time-consuming nature of the measurement and the expensive equipment required for the direct method $[3,33]$. This was clearly illustrated in a study of 535 adult patients undergoing RHC in whom resting oxygen uptake was assessed using estimated values according to three published formulae, or by direct assessment through timed collections of exhaled air via the Douglas bag technique [33]. Results from the study demonstrated a $>25 \%$ difference between directly measured and estimated values in $17-25 \%$ of patients, depending on the formula used to derive the estimated values [33].

\section{Technical errors and complications}

Aside from measurement or data interpretation errors, technical errors in the RHC procedure can manifest as respiratory, cardiac rhythm, hypotensive or device-related complications [34]. However, when performed in experienced centres with an established record of treating patients with $\mathrm{PH}$, RHC is associated with low complication and mortality rates [8, 34, 35]. A combined 5-year retrospective and 6-month prospective analysis of 7218 RHC procedures undertaken in 15 experienced centres in the USA and Europe reported an overall serious complication rate of only $1.1 \%$ and just four procedure-related

TABLE 2 Contraindications and complications associated with right heart catheterisation

Contraindications [36-38]

Complications ${ }^{\#}[34]$
Absolute: mechanical tricuspid or pulmonic valve, right heart masses (thrombus or tumour), and right-sided endocarditis

Relative: coagulopathy, pacemaker, bioprosthetic tricuspid or pulmonic valve, left bundle branch block, arrhythmias, and skin site infections

Haematoma at puncture sites, pneumothoraces, arrhythmias, vasovagal episodes, hypotensive episodes, and pulmonary haemorrhage

\#: the complications listed are those that occur most frequently. 
deaths (a rate of $0.06 \%$ ) among patients with $\mathrm{PH}$ [34]. Overall, the vast majority of complications were of mild-to-moderate severity and most commonly related to adverse events arising from venous access, vagal reactions or pulmonary vasoreactivity testing (table 2) [34].

Complications from RHC, while relatively infrequent, may be more likely to occur in patients with specific demographic or clinical characteristics [8,34]. A retrospective chart review of 1637 catheterisations performed over 10 years in 607 patients at a $\mathrm{PH}$ centre in the USA found that infants or toddlers aged $<2$ years experienced the highest risk of RHC-related complications, followed by patients who underwent general anaesthesia or a catheter-related intervention, or those who had an elevated mean RAP [8].

\section{RHC is crucial for avoiding misdiagnosis}

While RHC is associated with a low risk of complications, omitting the technique altogether from diagnostic assessment can increase the likelihood of misdiagnosis. However, if RHC is performed, incorrect measurement or interpretation of haemodynamic parameters may also give rise to diagnostic inaccuracies. Both of these scenarios can result in inappropriate treatment and care of a patient with PAH [3, 7]. The RePHerral study consecutively enrolled 140 newly diagnosed patients at an expert $\mathrm{PH}$ centre in order to determine the accuracy of diagnoses prior to referral [7]. The diagnosis at the referring centre was based on laboratory, pulmonary function, cardiac catheterisation and/or exercise capacity measures [7]. Overall, 25 (42\%) of the 59 patients who underwent RHC at the tertiary centre were found to have a diagnosis that differed from the initial diagnosis of $\mathrm{PH}$ received prior to referral [7]. Moreover, among the 56 patients diagnosed with PAH before referral, PAH was confirmed in 41 (73\%) while 7 (12\%) had no evidence of $\mathrm{PH}$ after tertiary care evaluation [7]. Notably, 42 (30\%) patients from the total study population had begun $\mathrm{PAH}$ treatments before referral, but, following tertiary centre evaluation, $57 \%$ of these patients were found to have been prescribed medications that are not supported by guideline recommendations [7].

\section{$P A H$ versus $P H$ due to heart failure with preserved ejection fraction}

Distinguishing between $\mathrm{PAH}$ and other types of $\mathrm{PH}$ presents a common challenge for interpretation of RHC haemodynamic measurements and can lead to potential errors in diagnosis [3, 14]. A frequent issue in the diagnostic work-up of $\mathrm{PAH}$ is differentiation from $\mathrm{PH}$ due to heart failure with preserved ejection fraction (HFpEF), as the presence of a normal PAWP value does not rule out HFpEF [3].

In volume-depleted patients with HFpEF or occult pulmonary venous hypertension, a normal PAWP accompanied by a high PAP can lead to an erroneous diagnosis of PAH [14, 39]. In this clinical scenario, a potential solution is fluid challenge during RHC, which can reduce the likelihood of misdiagnosis in patients with left ventricular dysfunction [3]. Fluid challenge during RHC has been explored to differentiate occult pulmonary venous hypertension from PAH $[40,41]$, defined as an increase in PAWP to $>15 \mathrm{mmHg}$ [39]. In a retrospective review of 207 patients meeting baseline haemodynamic criteria for $\mathrm{PAH}$, an increase in PAWP to $>15 \mathrm{mmHg}$ following fluid challenge with an intravenous fluid bolus of $0.5 \mathrm{~L}$ of normal saline over 5-10 min suggested diastolic left ventricular dysfunction in $22.2 \%$ of patients [39]. However, the use of exercise challenge in conjunction with RHC may enable a clearer distinction between HFpEF and other conditions given that exertional dyspnoea is a nonspecific symptom that does not readily differentiate early HFpEF [42]. Although growing evidence supports the validity of these techniques, further standardisation and evaluation are recommended before adopting fluid and exercise challenge in routine clinical practice [3].

There are a number of unrelated clinical conditions that can confound the interpretation of RHC data and affect the diagnosis of PAH, including advanced parenchymal lung or airway disease, or morbid obesity [14]. In the latter case, for example, intrathoracic pressure fluctuations are exaggerated, which can lead to an underestimation of the mean PAWP value $[14,43]$.

In summary, errors in measurement and data interpretation potentially encountered during RHC can increase the likelihood of misdiagnosis and complications. Minimising potential inaccuracies offers the opportunity to enhance the utility of RHC in the clinical setting.

\section{Evolving utilisation and application of RHC in clinical practice}

RHC is increasingly accepted and used in clinical practice, although utilisation rates and application of the procedure may vary by centre and by clinical specialty. The 2013 Pulmonary Arterial Hypertension Quality Enhancement Research Initiative reported high rates of RHC utilisation across academic (96.3\%) and community (84.4\%) centres [44]. However, there was some disparity in RHC use between the two types of centres (i.e. patients were less likely to have a diagnosis of PAH confirmed by RHC at a community centre), which suggests the need for greater adherence to clinical practice guideline recommendations [44]. In current practice, there are also differences in the utilisation of RHC by clinicians. For example, pulmonologists perform RHC as the sole invasive procedure, whereas cardiologists 
tend to use RHC in addition to other invasive methods, including left heart catheterisation. Moreover, the patient populations on whom RHC is performed are different, in that pulmonologists often see patients with chronic obstructive pulmonary disease, whereas patients with HFpEF are usually referred to cardiologists, although there is a high level of overlap or coexistence between these conditions.

Reaching a decision on when in the diagnostic pathway to perform RHC poses a challenge to both clinicians and healthcare resource managers. The fragile nature of patients with suspected PAH means that many centres are hesitant to perform invasive techniques such as RHC [8]. However, there are very few contraindications for RHC (table 2). Efforts are under way to develop clearer guidance on when to include RHC in the diagnostic pathway for patients with $\mathrm{PH}[3,45]$. As discussed, in the stepwise algorithm for $\mathrm{PH}, \mathrm{RHC}$ is included as the required diagnostic step to confirm PAH following physical examination and noninvasive screening using imaging tools, and prior to specific diagnostic tests to differentiate $\mathrm{PAH}$ from other conditions [3]. A new evidence-based detection algorithm for systemic sclerosis (SSc)-associated $\mathrm{PAH}$ incorporating noninvasive assessments and RHC as a mandatory final screening step was recently proposed based on data drawn from the cross-sectional, international DETECT study [45]. Ultimately, this algorithm may prevent a delay in the diagnosis of patients with SSc-associated PAH and increase the chance of detecting the disease at an earlier stage [45].

RHC remains the gold standard diagnostic technique, but multimodal noninvasive tests are currently used as a first step to ensure that RHC is not performed unnecessarily [10, 46-49]. The choice of which noninvasive test to use depends on factors such as guideline recommendations, institutional expertise, patient safety and convenience [46]. In particular, echocardiography, pulmonary function testing and ventilation/perfusion scans must be used in conjunction with RHC to secure an accurate diagnosis [3].

In addition to ensuring diagnostic accuracy, haemodynamic information obtained by RHC as an essential part of an array of clinical, invasive and noninvasive tests can also be used to guide treatment decisions and determine PAH severity and outcome $[3,4,6]$. Elevated RAP, low cardiac output/cardiac index, high PVR and low $\mathrm{SvO}_{2}$ at baseline, all of which are measures obtained from RHC, are predictors of poor outcomes in patients with PAH [23, 50-52]. In addition, repeat measurements of pulmonary haemodynamics by RHC, along with clinical information such as World Health Organization functional class status and noninvasive parameters, helps to assess the response to treatment and also carries prognostic information [4]. The ability of RHC to predict disease progression was recently demonstrated in specific PAH subtypes, such as SSc-associated PAH [53]. In a prospective, 4-year follow-up study that identified PAH in 89 (12\%) out of 722 patients with SSc using RHC, elevated mean RAP was reported to be the strongest haemodynamic predictor of mortality among the SSc-PAH group $(\mathrm{p}=0.0001)$ [53]. In addition, preliminary evidence suggests that exercise haemodynamics during RHC, as previously discussed, may be a more accurate predictor of long-term outcome than haemodynamic variables recorded at rest in patients with PAH $[6,54]$.

\section{Conclusions}

RHC remains the only technique for reliably and definitively diagnosing $\mathrm{PAH}$, and is associated with a low risk of serious complications. Notably, the potential exists for RHC-measured haemodynamic parameters to gauge response to therapy and confer prognostic value, thus guiding informed clinical decision-making and early therapeutic intervention. Despite clear recommendations on the use of RHC, best practice differs between centres. Furthermore, there is a clear need to apply the standardised protocols and to further improve standardisation to achieve optimal application of RHC, with resulting positive implications for diagnosis and earlier intervention for patients with $\mathrm{PAH}$ in routine clinical practice.

\section{Acknowledgements}

The authors would like to thank Anusha Bolonna from PAREXEL (Worthing, UK) for medical writing assistance, funded by Actelion Pharmaceuticals Ltd (Allschwil, Switzerland).

\section{References}

Chatterjee K. The Swan-Ganz catheters: past, present, and future. A viewpoint. Circulation 2009; 119: 147-152.

Nossaman BD, Scruggs BA, Nossaman VE, et al. History of right heart catheterization: 100 years of experimentation and methodology development. Cardiol Rev 2010; 18: 94-101.

3 Hoeper MM, Bogaard HJ, Condliffe R, et al. Definitions and diagnosis of pulmonary hypertension. J Am Coll Cardiol 2013; 62: Suppl., D42-D50.

4 Galiè N, Humbert M, Vachiery JL, et al. 2015 ESC/ERS Guidelines for the diagnosis and treatment of pulmonary hypertension. Eur Respir J 2015; 46: 903-975.

5 Simonneau G, Gatzoulis MA, Adatia I, et al. Updated clinical classification of pulmonary hypertension. J Am Coll Cardiol 2013; 62: Suppl., D34-D41.

6 Saggar R, Sitbon O. Hemodynamics in pulmonary arterial hypertension: current and future perspectives. Am J Cardiol 2012; 110: Suppl., 9S-15S.

7 Deano RC, Glassner-Kolmin C, Rubenfire M, et al. Referral of patients with pulmonary hypertension diagnoses to tertiary pulmonary hypertension centers: the multicenter RePHerral study. JAMA Intern Med 2013; 173: 887-893. 
8 Zuckerman WA, Turner ME, Kerstein J, et al. Safety of cardiac catheterization at a center specializing in the care of patients with pulmonary arterial hypertension. Pulm Circ 2013; 3: 831-839.

9 Rosenkranz S, Behr J, Ewert R, et al. Rechtsherzkatheter-Untersuchung bei pulmonaler Hypertonie [Right heart catheterization in pulmonary hypertension]. Dtsch Med Wochenschr 2011; 136: 2601-2616.

10 Grünig E, Peacock AJ. Imaging the heart in pulmonary hypertension: an update. Eur Respir Rev 2015; 24: 653-664.

11 Oudiz R, Langleben D. Cardiac catheterization in pulmonary arterial hypertension: an updated guide to proper use. Adv Pulm Hypertens J 2005; 4: 15-25.

12 Tsang T. Principles of echocardiography. In: Murphy JG, Lloyd MA, eds. Mayo Clinic Cardiology: Concise Textbook. 3rd Edn. Boca Raton, Mayo Clinic Scientific Press, 2008; pp. 117-142.

13 Howard C, Safdar Z. Pulmonary artery diastolic pressure gradient is an indicator of severity of illness in patients with pulmonary hypertension related to left-sided heart disease. Am J Respir Crit Care Med 2014; 189 : A3865.

14 Mathier M. The nuts and bolts of interpreting hemodynamics in pulmonary hypertension associated with diastolic heart failure. Adv Pulm Hypertens J 2011; 10: 33-40.

15 Grossman W. Blood flow measurement: cardiac output and vascular resistance. In: Baim DS, ed. Grossman's Cardiac Catheterization, Angiography, and Intervention. 7th Edn. Philadelphia, Lippincott Williams \& Wilkins, 2006; pp. 148-161.

16 Abtathian F, Jang I-K. Cardiac catheterization, coronary arteriography, and intravascular diagnostics. In: Gaggin HK, Januzzi JL, eds. MGH Cardiology Board Review. London, UK, Springer-Verlag, 2014; pp. 153-173.

17 Chatterjee NA, Lewis GD. Characterization of pulmonary hypertension in heart failure using the diastolic pressure gradient: limitations of a solitary measurement. JACC Heart Fail 2015; 3: 17-21.

18 Kovacs G, Berghold A, Scheidl S, et al. Pulmonary arterial pressure during rest and exercise in healthy subjects: a systematic review. Eur Respir J 2009; 34: 888-894.

19 Kovacs G, Olschewski A, Berghold A, et al. Pulmonary vascular resistances during exercise in normal subjects: a systematic review. Eur Respir J 2012; 39: 319-328.

20 Kovacs G, Avian A, Pienn M, et al. Reading pulmonary vascular pressure tracings. How to handle the problems of zero leveling and respiratory swings. Am J Respir Crit Care Med 2014; 190: 252-257.

21 Lavdaniti M. Invasive and non-invasive methods for cardiac output measurement. Int J Caring Sciences 2008; 1 : $112-117$

22 Miller A, Soukoulis V, Lilly LS. Section I. Fundamentals of cardiovascular disease; genetics and personalized medicine; evaluation of the patient. In: Lilly LS, ed. Braunwald's Heart Disease. Review and Assessment. 9th Edn. Philadelphia, Elsevier Saunders, 2012; pp. 1-86.

23 Wensel R, Francis DP, Meyer FJ, et al. Incremental prognostic value of cardiopulmonary exercise testing and resting haemodynamics in pulmonary arterial hypertension. Int J Cardiol 2013; 167: 1193-1198.

24 Rich S, Kaufmann E, Levy PS. The effect of high doses of calcium-channel blockers on survival in primary pulmonary hypertension. N Engl J Med 1992; 327: 76-81.

25 Sitbon O, Humbert M, Jais X, et al. Long-term response to calcium channel blockers in idiopathic pulmonary arterial hypertension. Circulation 2005; 111: 3105-3111.

26 Montani D, Savale L, Natali D, et al. Long-term response to calcium-channel blockers in non-idiopathic pulmonary arterial hypertension. Eur Heart J 2010; 31: 1898-1907.

27 Oliveira EC, Amaral CF, Moura MA, et al. Testing pulmonary vasoreactivity. J Bras Pneumol 2008; 34: 838-844.

28 Farber HW, Gibbs S. Under pressure: pulmonary hypertension associated with left heart disease. Eur Respir Rev 2015; 24: 665-673.

29 Tonelli AR, Mubarak KK, Li N, et al. Effect of balloon inflation volume on pulmonary artery occlusion pressure in patients with and without pulmonary hypertension. Chest 2011; 139: 115-121.

30 LeVarge BL, Pomerantsev E, Channick RN. Reliance on end-expiratory wedge pressure leads to misclassification of pulmonary hypertension. Eur Respir J 2014; 44: 425-434.

31 Ryan JJ, Rich JD, Thiruvoipati T, et al. Current practice for determining pulmonary capillary wedge pressure predisposes to serious errors in the classification of patients with pulmonary hypertension. Am Heart J 2012; 163: 589-594.

32 Hansdottir S, Groskreutz DJ, Gehlbach BK. WHO's in second?: A practical review of World Health Organization group 2 pulmonary hypertension. Chest 2013; 144: 638-650.

33 Narang N, Thibodeau JT, Levine BD, et al. Inaccuracy of estimated resting oxygen uptake in the clinical setting. Circulation 2014; 129: 203-210.

34 Hoeper MM, Lee SH, Voswinckel R, et al. Complications of right heart catheterization procedures in patients with pulmonary hypertension in experienced centers. J Am Coll Cardiol 2006; 48: 2546-2552.

35 Ranu H, Smith K, Nimako K, et al. A retrospective review to evaluate the safety of right heart catheterization via the internal jugular vein in the assessment of pulmonary hypertension. Clin Cardiol 2010; 33: 303-306.

36 Gupta D, Karrowni W, Chatterjee K. Swan-Ganz catheters. In: Chatterjee K, Anderson M, Heistad D, et al., eds Cardiology: An Illustrated Textbook. New Delhi, Jaypee Brothers Medical Publishers Ltd, 2012; pp. $503-516$.

37 Martin UJ, Krachman S. Hemodynamic monitoring. In: Criner GJ, D’Alonzo GE, eds. Critical Care Study Guide: Text and Review. Philadelphia, Springer-Verlag, 2002; pp. 44-69.

38 Kern MJ, Sorajja P, Lim M. The Cardiac Catheterization Handbook. 6th Edn. Oxford, Elsevier Health Sciences, 2015

39 Robbins IM, Hemnes AR, Pugh ME, et al. High prevalence of occult pulmonary venous hypertension revealed by fluid challenge in pulmonary hypertension. Circ Heart Fail 2014; 7: 116-122.

40 Fujimoto N, Borlaug BA, Lewis GD, et al. Hemodynamic responses to rapid saline loading: the impact of age, sex, and heart failure. Circulation 2013; 127: 55-62.

41 Frost AE, Farber HW, Barst RJ, et al. Demographics and outcomes of patients diagnosed with pulmonary hypertension with pulmonary capillary wedge pressures 16 to $18 \mathrm{~mm} \mathrm{Hg}$ : insights from the REVEAL Registry. Chest 2013; 143: 185-195.

42 Borlaug BA, Nishimura RA, Sorajja P, et al. Exercise hemodynamics enhance diagnosis of early heart failure with preserved ejection fraction. Circ Heart Fail 2010; 3: 588-595.

43 Boerrigter $\mathrm{BG}$, Waxman $\mathrm{AB}$, Westerhof $\mathrm{N}$, et al. Measuring central pulmonary pressures during exercise in COPD: how to cope with respiratory effects. Eur Respir J 2014; 43: 1316-1325. 
McLaughlin VV, Langer A, Tan M, et al. Contemporary trends in the diagnosis and management of pulmonary arterial hypertension: an initiative to close the care gap. Chest 2013; 143: 324-332.

45 Coghlan JG, Denton CP, Grunig E, et al. Evidence-based detection of pulmonary arterial hypertension in systemic sclerosis: the DETECT study. Ann Rheum Dis 2014; 73: 1340-1349.

46 Bossone E, Dellegrottaglie S, Patel S, et al. Multimodality imaging in pulmonary hypertension. Can J Cardiol 2015; 31: 440-459.

47 Constantinescu T, Magda SL, Niculescu R, et al. New echocardiographic tehniques in pulmonary arterial hypertension vs. right heart catheterization - a pilot study. Maedica (Buchar) 2013; 8: 116-123.

48 Fisher MR, Forfia PR, Chamera E, et al. Accuracy of Doppler echocardiography in the hemodynamic assessment of pulmonary hypertension. Am J Respir Crit Care Med 2009; 179: 615-621.

49 El-Korashy RIM, Amin YM, Eissa AI, et al. Echocardiography versus right heart catheterization in class I pulmonary hypertension. Egypt J Chest Dis Tuberc 2014; 63: 419-422.

50 Humbert M, Sitbon O, Yaici A, et al. Survival in incident and prevalent cohorts of patients with pulmonary arterial hypertension. Eur Respir J 2010; 36: 549-555.

51 Nickel N, Golpon H, Greer M, et al. The prognostic impact of follow-up assessments in patients with idiopathic pulmonary arterial hypertension. Eur Respir J 2012; 39: 589-596.

52 Benza RL, Miller DP, Gomberg-Maitland M, et al. Predicting survival in pulmonary arterial hypertension: insights from the Registry to Evaluate Early and Long-Term Pulmonary Arterial Hypertension Disease Management (REVEAL). Circulation 2010; 122: 164-172.

53 Mukerjee D, St George D, Coleiro B, et al. Prevalence and outcome in systemic sclerosis associated pulmonary arterial hypertension: application of a registry approach. Ann Rheum Dis 2003; 62: 1088-1093.

54 Chaouat A, Sitbon O, Mercy M, et al. Prognostic value of exercise pulmonary haemodynamics in pulmonary arterial hypertension. Eur Respir J 2014; 44: 704-713. 